\title{
Application of genetic algorithm in target image processing of TV guided missile
}

\author{
Jing Feng He, Ru Liang Xiao, Ming Ji and Guang Zhen Zhang \\ College of Control Engineering, Xijing University, NO.1 Xijing Road, Xian,China
}

\begin{abstract}
Target image processing requirements of the TV guided missile is high, and the speed is fast, and the quality of target image segmentation is high.In this paper, an improved genetic algorithm based on genetic algorithm is introduced, which is used in the image segmentation of the TV guided missile, and the processing effect is good and the calculation speed is fast.Simulation results show that the algorithm is effective.
\end{abstract}

KeyWord.Image segmentation; Inter class variance method; Genetic algorithm

\section{PREFACE}

TV guided missile is widely used in one aspect of attack of air-to-ground because of having the characteristics of flexibility, rapid response, without geographical influence.After missile seeker intercepts the target ,Its track starts to work, and Calculates on the image to complete real-time tracking of the target. The segmentation of target directly impact on identification and track of target,so the study on segmentation of target is of great significance to combat effects of TV guided missile.

\section{DEVELOPMENT STATUS OF THE IMAGE SEGMENTED METHOD}

At present,there are many methods of image segmentation, and common segmented method consists of edge detection type,regional tracking,threshold type $^{[1]}$.Threshold type is a method that is simple and effective,simple to calculation, fast,and is a preferred method for image processing,Because the TV-guided missiles in recognition of the target tracking has a high demand on quality, speed and unity of the divided regions of target image segmentation.In this paper,we using inter class variance method based on high quality image segmentation in Threshold as foundation, and propose an improved Inter class variance method based on genetic algorithm .

\section{AN IMPROVED INTER CLASS VARIANCE METHOD BASED ON GENETIC ALGORITHM}

\subsection{The principle upon image segmentation of threshold type}

Order the grayscale range of the image that captured by seeker as $G=\{0,1, \ldots, l-1\}, t \in G$ as a limit, ${ }^{B}=\left\{b_{0} b_{1}\right\}$ as one pair of binary gray,and $b_{0} b_{1} \in G$.The threshold settings of $\mathrm{t}$ can be obtained by a binary image of $\mathrm{f}_{\mathrm{t}}$, that is,

$$
f_{t}(x, y)= \begin{cases}b_{0} & f(x, y)<t \\ b_{1} & f(x, y) \geq t\end{cases}
$$

Threshold method of image segmentation is a process in accordance with the nor that select the most appropriate $t$ in the range of possible values. Threshold method is divided into global threshold and local threshold. Global threshold to the whole image only sets a threshold segmentation, however, local threshold to the whole image is divided into several sub-graph, and each sub is set a threshold of segmentation.Local threshold has been more widely used, and inter-class variance method (Otsu) is one kind of representative of algorithm in local threshold.

\subsection{The basic principle of inter class variance method}

Threshold setting of inter class variance method divides image into target class $\mathrm{C}_{0}$ and background class $\mathrm{C}^{1[2]}$.and $c_{0}=\{0,1, \ldots ., t\} \quad c_{1}=\{t+1, \ldots ., l-1\}{ }_{\text {.Set }} \sigma_{b}^{2}\left(t^{*}\right)$ as inter class variance method, and the choice of optimum threshold $t^{*}$ is the gray level which the maximum of $\sigma_{b}^{2}$ is corresponding to. This is:

$$
\sigma_{b}^{2}\left(t^{*}\right)=\max _{t=G} \sigma_{b}^{2}(t)
$$




$$
\begin{aligned}
& \text { among } \\
& \begin{array}{c}
\sigma_{b}^{2}(t)=\omega_{0}\left(m_{0}-m\right)^{2}+\omega_{1}\left(m_{1}-m\right)^{2} \\
=\omega_{0} \omega_{1}\left(m_{1}-m_{0}\right)^{2}
\end{array} \\
& \begin{array}{c}
\omega_{0}=\operatorname{Pr}\left(c_{0}\right)=\sum_{i=0}^{t} p_{i} \\
\omega_{1}=\operatorname{Pr}\left(c_{1}\right)=\sum_{i=t+1}^{l-1} p_{i}=1-\omega_{0} \\
m_{0}=\sum_{i=0}^{t} i \operatorname{Pr}\left(c_{0} \mid i\right)=\sum_{i=0}^{t} i p_{i} / \omega_{0} \\
m_{1}=\sum_{i=t+1}^{l-1} i \operatorname{Pr}\left(c_{1} \mid i\right)=\sum_{i=t+1}^{l-1} i p_{i} / \omega_{1} \\
m=\sum_{i=0}^{l-1} i p_{i}
\end{array}
\end{aligned}
$$

Pi the rate of occurrence of Grayscale $i$ and the graph of $i$ are called gray histogram.

\subsection{Concrete realization of of improved Otsu}

Otsu method will eventually need to determine the optimal threshold value $t^{*}$ to make the $\sigma_{b}^{2}$ biggest.How to choose $t^{*}$ directly relate to the quality of image segmentation.For TV-guided missile, its onboard computer speech is slow, but seeker has higher requirement on the target image. The original method of optimization is not only time consuming but also requires a lot of math, not suitable for use in TV-guided missile,therefore, we need a fast and efficient method to find the optimal threshold.Based on this request, we propose the genetic algorithm optimization algorithm is introduced into the Ostu, in search of a suitable space, use a genetic algorithm to find the optimal value ${ }^{[3]}$.

Genetic algorithm ${ }^{[4]}$ (Genetic Algorithm abbreviated GA)is a new kind of simulation of the evolution of life mechanism and optimization , and is a optimization equation that is with the natural genetics and computer science.It is a probabilistic search algorithm, uses some coding technique called chromosome acting on index string and simulate evolutionary process of group including these strings, using some kind of effect on the index string encoding technique called chromosome evolution simulation string composed from these groups, in order to find the best individual that suitable for a specific problem.

In our algorithm, we must first determine the decision variables and the various constraints that determine the individual's phenotype $\mathrm{X}$ and solution space of problem.Decision variables are the optimal threshold required of $t^{*}$.The image captured by TV-guided missile seeker of is a 256 grayscale image $^{[5]}$, and each pixel is an integer, and the range is 0 to 255 ,so the optimal threshold should be within this range, and is integer.Second, the optimal model, which determines the form of the mathematical description of the objective function and quantification methods. The specific design of fitness function uses the value of $\sigma_{b}^{2}$ as the standard, and the theory of genetic algorithms are generally established on the basis of looking at the minimum, therefore, function equation of $\sigma_{b}^{2}$ should be transformed, this is: order

$g(t)=-\sigma_{b}^{2}(t)=-\omega_{0} \omega_{1}\left(m_{1}-m_{0}\right)^{2}$

Since $\sigma_{b}^{2}(t)$ is a non-positive, we transform $g(t)$ into minimum issue.Third, determine to represent the coding method of feasible solution of chromosome. For the coding of optimal threshold $t^{*}$ can represent binary coded 0,1 , its value ranges from 0 to 255 , with eight of the code strings, ie 00000000-1111111. Fourth, Fourth, determine the method of quantitative evaluation of individual fitness. Since $\sigma_{b}^{2}(t)$ is a non-positive,So take the minimum individual of $g(t)$ as criteria. Fifth, design genetic operator and designing the specific operating methods of determining Selection operator, crossover operator, mutation operator.In order to meet the onboard computer and seeker needs to achieve better segmentation results in the shortest possible time in our algorithm ,So we choose the simpler operator in terms of operator.Wherein the selection operation to select proportional selection operator, crossover operator to select a single point of crossover, probability is set to 0.8 , mutation operation selection uniform mutation (Uniform Mutation) operator, the probability is set to 0.008 .Sixth,to determine the operating parameters relating to genetic algorithms and stop criterion.In the calculation taking into account the impact of the onboard computer speed,and evolution algebra and a large population can not be set, we taken 20,10. Stop algebraic criterion set an upper limit, namely stopping operation when reach the maximum algebraic.

Experiments show that the use of this class of genetic algorithm can get the global optimal solution of the optimal threshold $t^{*}$ in a very short time. This fully meets the speed requirements onboard computer, and a better image segmentation.

\section{The simulation analysis}

The experiment is completed on the ground simulated projectile of TV-guided missile.First,we through seeker to capture two images of ground targets, which is transmitted to the onboard computer as an image segmentation original picture, and then by the onboard computer for image processing, image process after the original image and are fed to the type ground surveillance computer simulated projectile. The following is a target image of the original image of ground targets and the use of based on improved genetic algorithm between class variance law division. 


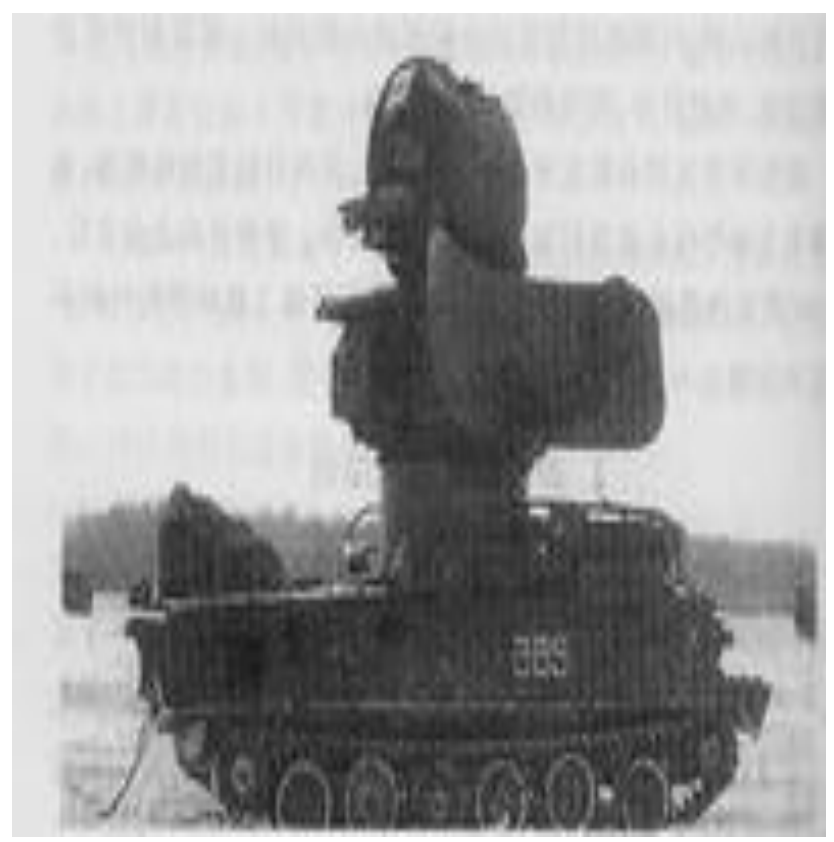

(a) Goal one artwork

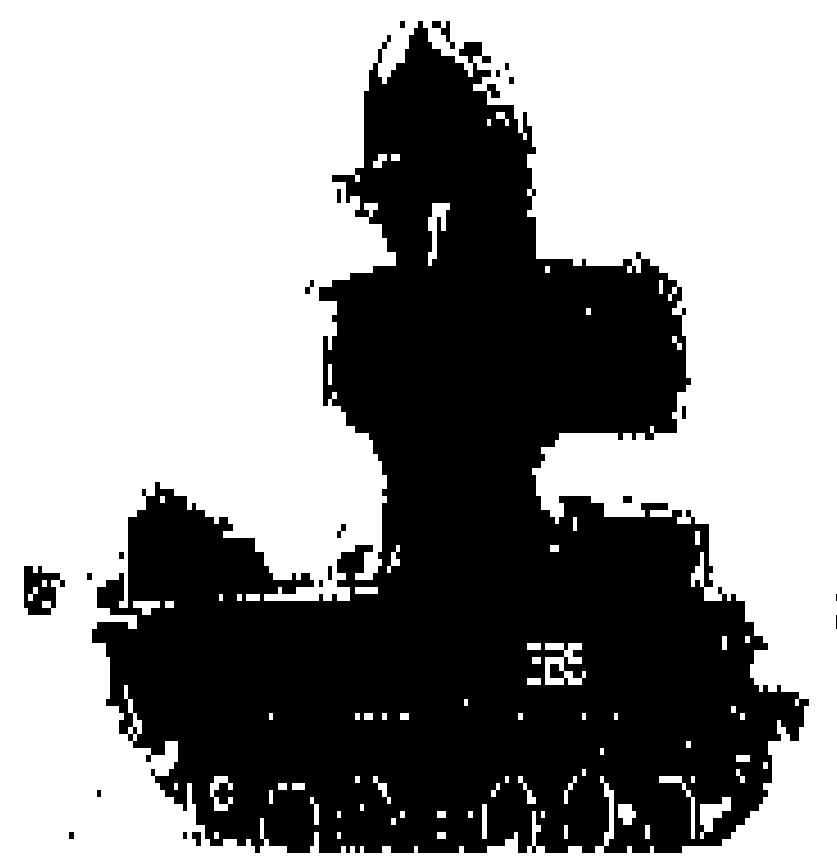

(b) Goal two segmented image

Figure I target- image segmentation

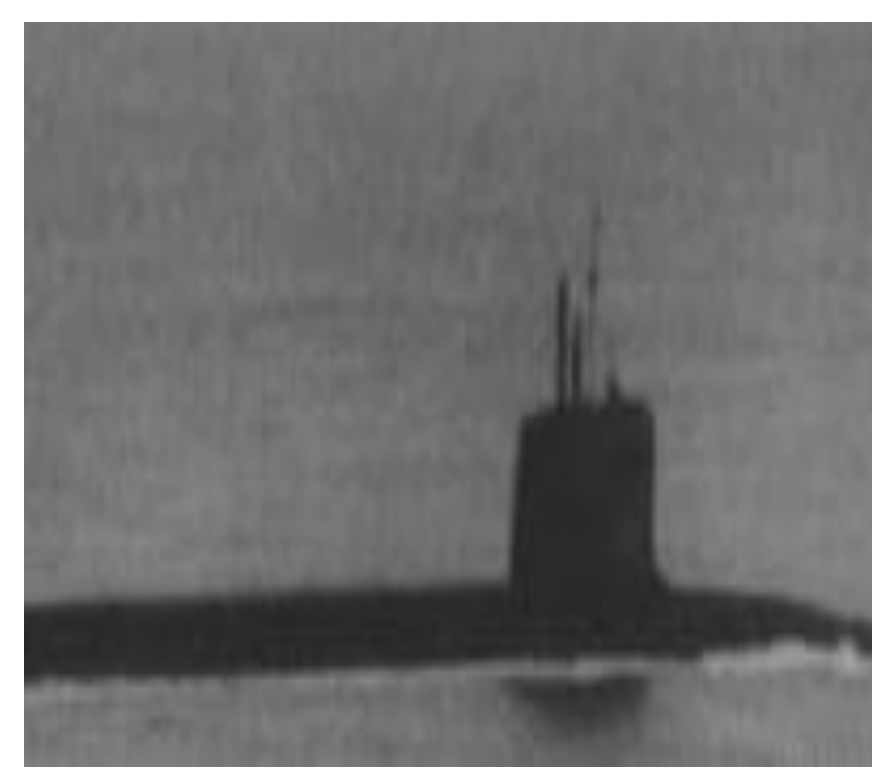

(a) Goal two artwork

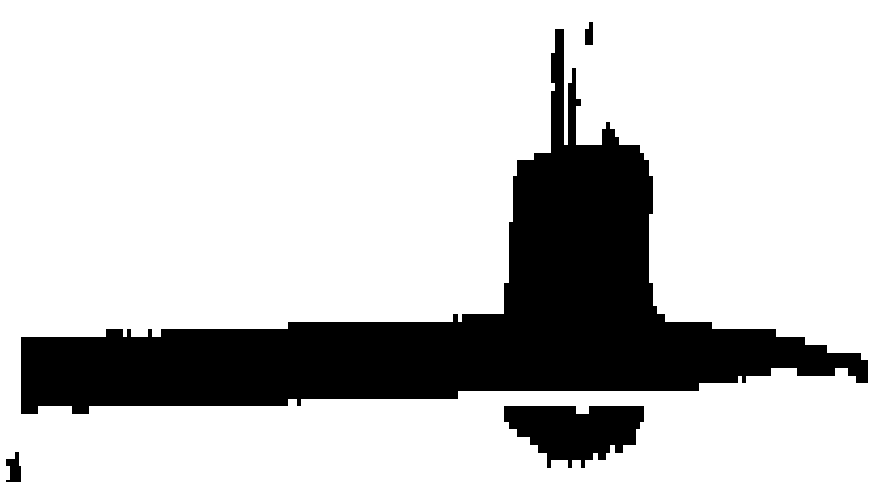

(b) Goal two segmented image

Figure 2 target- image segmentation

From the segmented image can be seen, two target image used inter class variance method have a good segmentation results. The target is clearly separated from the background, and the basic characteristics of the target is also well demonstrated.

\section{Conclusion}

Experiments show that, based on the type of TVguided missiles of this approach can distinguish, track targets very good and always with the goal keep the range of allowable deviation. This shows that the target image segmentation In this method not only has a good segmentation effect, while the requirements for the onboard computer of the TV-guided missile, which provides a good research methods in the study of television guided missiles in. 


\section{References}

[1]Zhon Ming,Sun Shudong.The principle and application of the genetic algorithm [M] 。 Beijing:national defense industry press, 2002

[2]Hou Gexian.Genetic algorithm and its application in tracking system [D],Xi'an: Xi'an university of electronic science and technology, 1998

[3]Cui Yi.The technology and application of digital image process[M].Beijing:electronic industry press,2001

[4]Peng Xuan.Genetic algorithm and its application in image segmentation [D].Changchun: Jilin university graduate thesis, 2006

[5]Le Zhongxin.Digital image process [M] Beijing: national defense industry press, 2003. 